\title{
Blindzonenfreie Ultraschall-Abstandsmessung mit codierten Sendesignalen
}

\author{
Schröder, Andreas; Henning, Bernd \\ Universität Paderborn EIM-E, Elektrische Messtechnik, Warburger Str. 100, 33098 Paderborn \\ Tel.: +49 5251 60-3018, E-Mail: schroeder@emt.upb.de
}

\section{Kurzfasssung}

Bei der Ultraschall-basierten Abstandsmessung mit einem einzelnen Schallwandler ergibt sich durch das Prinzip bedingt eine Blindzone. Während und unmittelbar nach der Anregung durch das Sendesignal ist es im Allgemeinen nicht möglich, das Empfangssignal am Ultraschallwandler auszuwerten. Eine Verwendung von codierten Sendesignalen (z.B. zur Unterscheidung verschiedener Sensoren) verlängert diesen Blindbereich zusätzlich.

Dieser Beitrag zeigt eine Möglichkeit zur Realisierung eines simultanen Sende- und Empfangsbetriebs mit einem einzelnen Schallwandler. Dadurch wird es möglich, die Blindzone auch bei sehr langen Sendesignalen zu eliminieren. Dazu wird der Ultraschallwandler über einen Vorwiderstand betrieben und dessen Spannung gemessen. Mittels eines mathematischen Modells kann das elektrische Sendesignal, welches am Ultraschallwandler anliegt, bestimmt werden. Die Subtraktion dieses Signals von der gemessenen Schallwandlerspannung liefert das elektrische Empfangssignal.

Aufgrund von z.B. Temperatureinflüssen ändern sich die elektrischen Eigenschaften des Ultraschallwandlers ständig, wodurch eine Anpassung des Modells notwendig wird. Daher kann die Modellidentifikation nicht im Vorfeld mit einer Freifeldmessung erfolgen, sondern ist permanent während des Betriebes notwendig. Tritt dabei ein Empfangssignal z.B. infolge einer Reflexion auf, so wird die Bestimmung der Modellparameter gestört. Durch eine Bewertung der ermittelten Modelle kann die Robustheit einer Abstandsmessung erhöht werden.

\section{Einleitung}

Viele Ultraschall-basierte Sensoren für die Abstandsmessung in Luft arbeiten mit einem einzelnen Schallwandler. Dies vereinfacht die mechanische Konstruktion und maximiert die für das Senden und Empfangen nutzbare aktive Fläche. Allerdings folgen hierraus auch Nachteile. Ein Empfangssignal kann erst nach dem Ausschwingen des Schallwandlers erfasst werden. Daraus resultiert bei der Abstandsmessung eine Blindzone in der nicht gemessen werden kann. Diese Blindzone kann in gewissen Grenzen durch die Betriebsart (z.B. Länge oder Frequenz des Sendesignals) beeinflusst werden. Aufgrund der hohen Güte und geringen Bandbreite des Schallwandlers kann das Sendesignal allerdings nicht beliebig kurz erzeugt werden. Ebenso kann die Sendefrequenz nicht beliebig hoch gewählt werden, da die Dämpfung in der Luft mit steigender Frequenz zunimmt [1] und somit den maximal messbaren Abstand limitiert. Somit ist für das Sensordesign ein Kompromiss zwischen geringer Blindzone und großem Erfassungsbereich zu wählen.

Sollen mehrere Ultraschallsensoren gleichzeitig in einer Umgebung arbeiten, ohne sich gegenseitig zu beeinflussen, besteht die Möglichkeit, diese untereinander zu synchronisieren, um sicherzustellen, dass keine Signale anderer Sensoren erfasst und ausgewertet werden. Ist eine Synchronisation nicht möglich (z.B. bei mobilen Robotern u.a.), können auch codierte Sendesignale eingesetzt werden, was bei herkömmlichen Abstandssensoren aufgrund der größeren Sendesignallänge allerdings zu einer deutlichen Vergrößerung der Blindzone führt.

Eine mögliche Lösung zur Reduzierung der Blindzone bildet der simultane Sende- und Empfangsbetrieb. Dabei wird mit verschiedenen Ansätzen versucht, das Sende- und Empfangssignal zu trennen [2, 3, 4, 5]. Das hier verwendete Verfahren basiert auf einer modellgestützten Bestimmung des Empfangssignals. Dazu wird der Schallwandler, wie in Abbildung 1 dargestellt, über einen definierten Vorwiderstand $R_{V}$ betrieben.

Die am Schallwandler messbare Spannung $u_{\mathrm{W}}$ setzt sich dann aus dem elektrischen Sendesignal $u_{\mathrm{WG}}$, das von dem Generator erzeugt wird, und dem elektrischen Empfangssignal $u_{\mathrm{E}}$ zusammen. Die Bestimmung des gesuchten elektrischen Empfangssignals erfolgt im Betrieb durch eine Subtraktion eines 
berechneten Kompensationssignals, welches aus dem Generatorsignal unter zu Hilfenahme eines Schallwandlermodells bestimmt wird, vom digitalisierten Schallwandlersignal. Im einfachsten Fall kann das Schallwandlermodell bei einer Freifeldmessung ermittelt werden. Allerdings muss das Modell bei sich ändernden Umweltbedingungen (z.B. einer Temperaturänderung), angepasst werden, so dass kein Modell mit fixen Parametern verwendet werden kann.

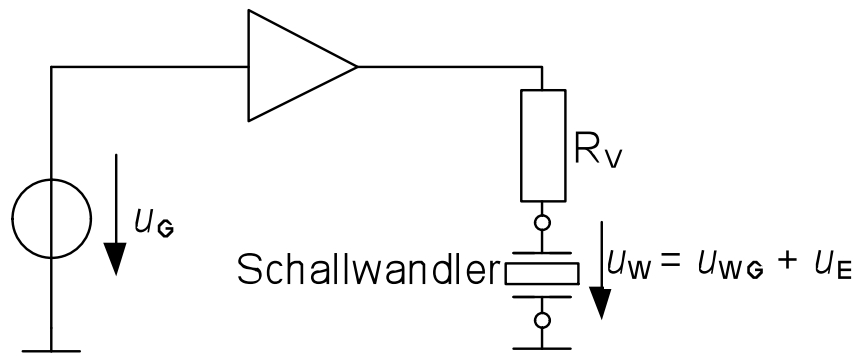

Abbildung 1: Prinzipielle Beschaltung des Schallwandlers

Zur Nachführung der Modellparameter kann z.B. ein iterativer Ansatz, wie in [6] gezeigt wird, verwendet werden. Problematisch ist allerdings der Zeitpunkt nach dem Einschalten des Sensors. Hier stehen nur sehr wenige Messungen für die Identifikation des Schallwandlermodells zur Verfügung. Ein möglicher Ansatz zur Lösung dieses Startproblems ist im Folgenden beschrieben.

\section{Messaufbau}

Der verwendete Messaufbau besteht aus einem USB-Oszilloskop mit integriertem Sendesignalgenerator. Als Schallwandler kommt ein Bimorph-Luftultraschallwandler vom Typ 400ST160 von Pro-wave Electronics Corp. zum Einsatz, der eine Mittenfrequenz von etwa $40 \mathrm{kHz}$ und eine Bandbreite von $2 \mathrm{kHz}$ besitzt. Der Generator steuert den Schallwandler über einen Leistungsverstärker, der den Vorwiderstand $\mathrm{R}_{\mathrm{V}}$ beinhaltet, an. Der Verstärker basiert auf einem Operationsverstärker vom Typ THS6012. Dabei werden sowohl das Generatorsignal $u_{\mathrm{G}}$ als auch das Schallwandlersignal $u_{\mathrm{W}}$ vom Oszilloskop aufgezeichnet und anschließend in MATLAB® weiterverarbeitet. Die Abtastfrequenz beträgt 1,5625 MHz und die Quantisierung 14 Bit. Die Triggerung des Gesamtsystems erfolgt ebenfalls über den Generator. Der Schallwandler ist, wie in Abbildung 3 dargestellt, auf einem automatisiert fahrbaren Schlitten vor einem festen Metallreflektor (100x100 mm) montiert. Für die Erzeugung der Messdaten wird der Schlitten mit einer Schrittweite von $1 \mathrm{~mm}$ in einem Abstand von $0 \mathrm{~mm}$ bis $600 \mathrm{~mm}$ verfahren. So werden 601 Messungen mit verschiedenen Reflektorabständen erzeugt.

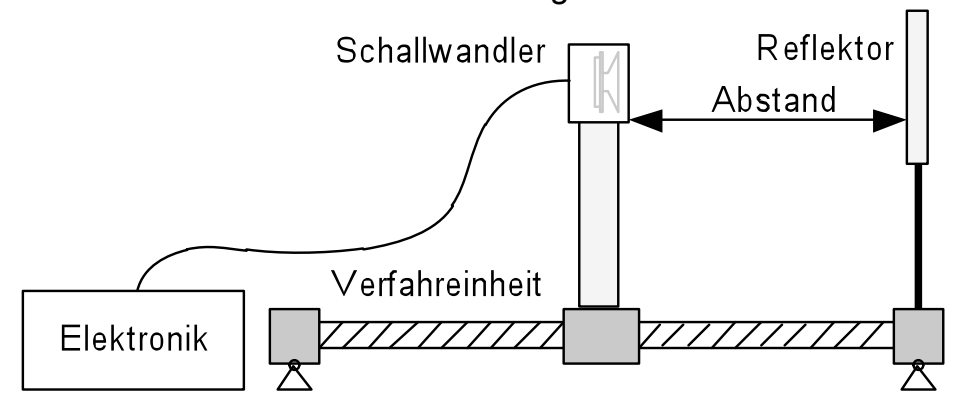

Abbildung 3: Mechanischer Versuchsaufbau

\section{Sendesignal und Schallwandlersignal}

Um die Sendesignale mehrerer Sensoren unterscheiden zu können, werden nun codierte Sendesignale genutzt. Dafür eignen sich Gold-Codes besonders gut, da sie untereinander orthogonal sind, also eine sehr geringe Kreuzkorrelation besitzen [7]. Weiterhin sind sie aufgrund ihres ausgeprägten Maximums bei der Autokorrelation gut für die Laufzeitbestimmung mittels Korrelation geeignet. Die hier genutzten GoldCodes besitzen eine Länge von 31 Bit. Damit ist es möglich, 33 verschiedene Codesequenzen zu erzeugen. Als Modulation wird eine binäre Phasenmodulation (BPSK) mit einer Symbollänge von vier Wellenzügen genutzt, was zu den in Abbildung 5 dargestellten zeitlichen Verläufen des Generator- und Schallwandlersignals führt. 


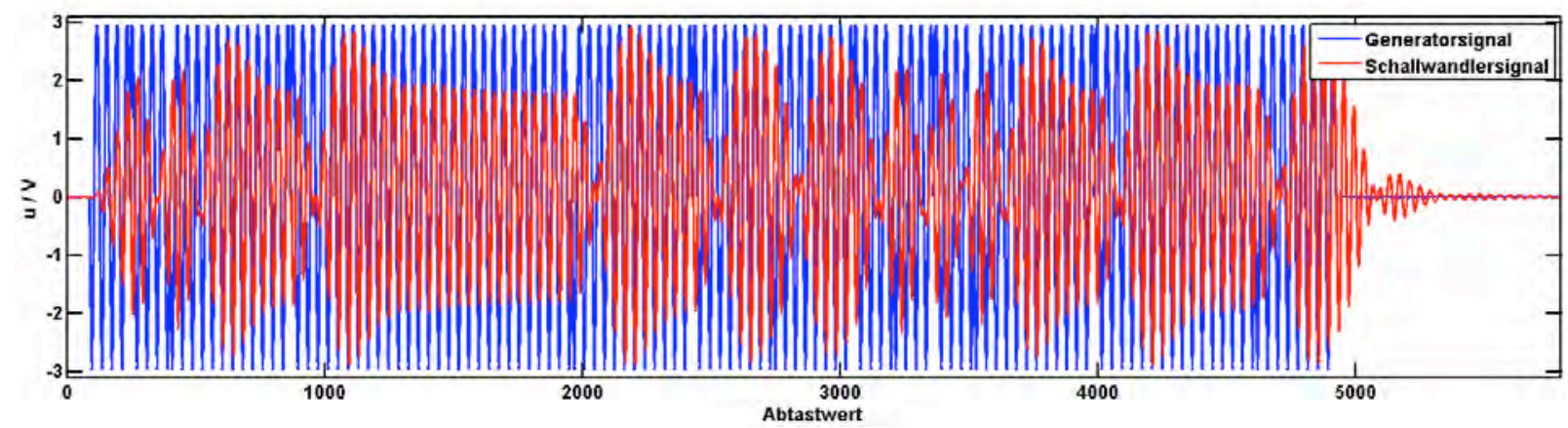

Abbildung 5: Generator- und Schallwandlersignal ohne Echo bei einer ausgewählten Codesequenz

\section{Empfangssignal}

Die Empfangssignalamplitude hängt von mehreren Einflüssen, wie z.B. der Schalldämpfung in der Luft, dem Divergenzwinkel, dem elektroakustischen Koppelfaktor des Schallwandlers sowie den Reflektoreigenschaften ab. Die Schalldämpfung in Luft liegt bei einer Frequenz von $40 \mathrm{kHz}$ etwa bei $1,3 \mathrm{~dB} / \mathrm{m}$. Der eingesetzte Schallwandler (400ST160) besitzt einen relativ großen Divergenzwinkel von $27^{\circ}$, wie die Messung in Abbildung 7 zeigt.

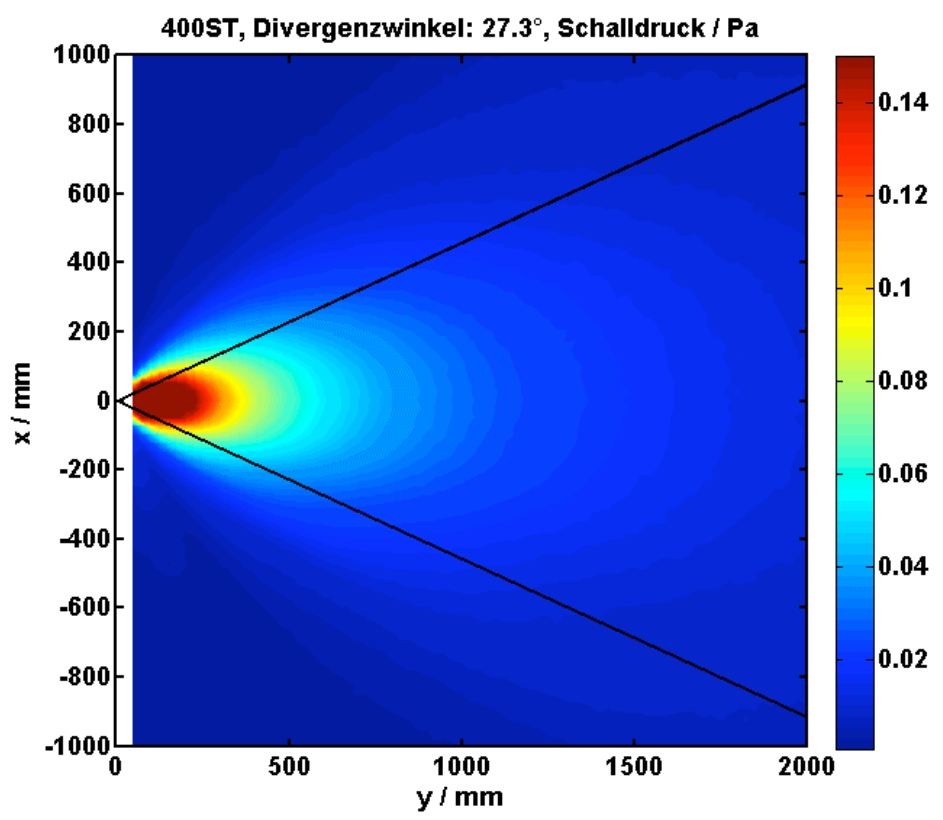

\section{Abbildung 7: Schallfeld des verwendeten Schallwandlers (400ST160); (Farblegende: Schallwechseldruck in $\mathrm{Pa}$, lineare Darstellung)}

Zur Abschätzung der zu erwartenden Empfangssignalamplitude erfolgt eine Messung mit einem zweiten nahezu identischen Schallwandler. Dazu wird der Reflektor im Messaufbau durch diesen zweiten Schallwandler ersetzt und dessen Empfangssignal für verschiedene Abstände von 0 mm bis $1950 \mathrm{~mm}$ mit $1 \mathrm{~mm}$ Schrittweite gemessen. Die gemessene, abstandsabhängige relative Empfangssignalamplitude (bezogen auf das Maximum der elektrischen Sendesignalamplitude) ist in Abbildung 9 dargestellt. Hier sind die Ergebnisse von zwei Messungen gegenübergestellt, eine mit BPSK moduliertem Sendesignal und eine mit einem Sinusburst mit 30 Wellenzügen als Sendesignal. Die geringere

Empfangssignalamplitude der Messung mit dem BPSK moduliertem Sendesignal ist durch die geringe Bandbreite des Schallwandlers begründet. Bei den gewählten Symbollängen schwingt dieser nie vollständig ein und erreicht daher nie den maximal möglichen Schallwechseldruck. Aus dem Verlauf der Empfangssignalamplituden lässt sich ableiten, wie hoch die Sendesignalunterdrückung für einen bestimmten Reflektorabtand mindestens sein muss, damit das Empfangssignal in der gleichen Größenordnung wie das Sendesignal liegt und durch eine Kreuzkorrelation fehlerfrei erkannt werden kann. 
Das in Abbildung 5 dargestellte Sendesignal besitzt 5500 Abtastwerte, was einer Dauer von 3,5 ms entspricht. Daraus ergibt sich, dass das ausgesandte Schallsignal eine Strecke von 1,2 m zurückgelegt hat, bis das Anregesignal am Wandler nahezu vollständig abgeklungen ist. Somit ist im schlechtesten Fall eine Sendesignalunterdrückung (entspricht dem Verhältnis des Maximums des berechneten Empfangssignals zum Maximum des Schallwandlersignals bei einer Freifeldmessung) von $53 \mathrm{~dB}$ notwendig, um das Empfangssignal zuverlässig identifizieren zu können. Bei größeren Entfernungen ist die Sendesignalunterdrückung unkritisch, da sich Sende- und Empfangssignal nicht mehr überlagern.

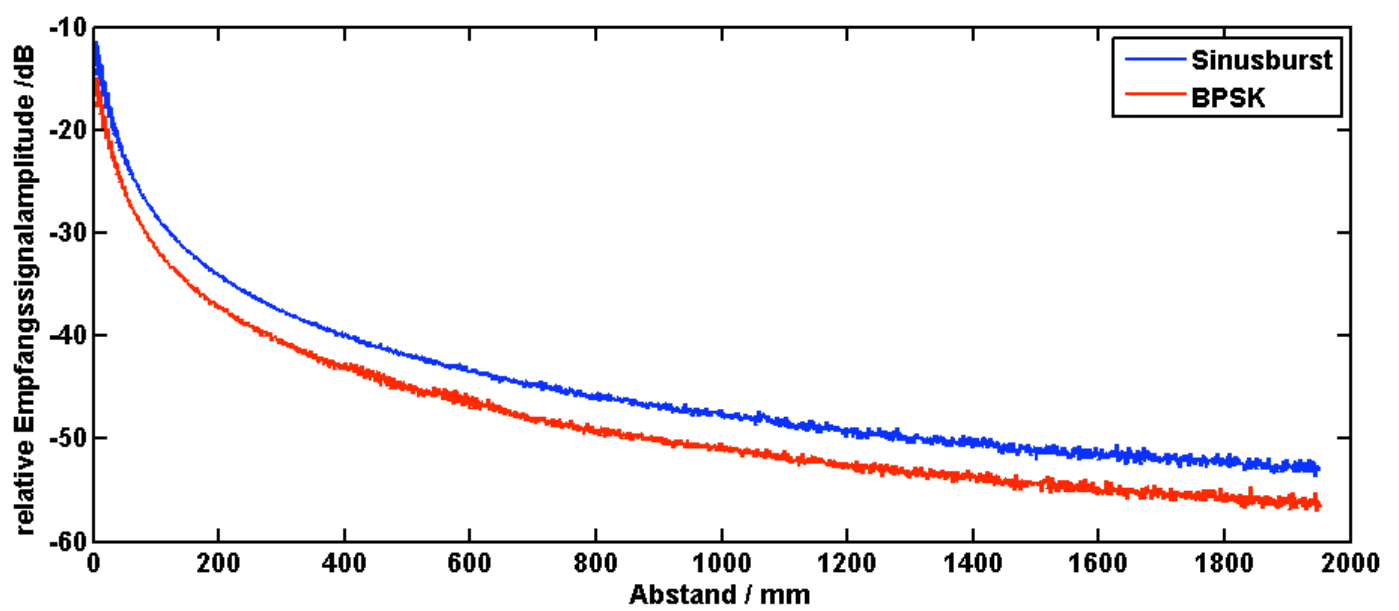

\section{Abbildung 9: Abstandsabhängigkeit der relativen Empfangssignalamplitude bei Anregung mit einem Sinusburst und einem BPSK-Signal}

\section{Modellidentifikation}

Als mathematisches Modell für den Schallwandler mit Vorwiderstand wird ein IIR-Filter genutzt, dessen Parameter mittels des StMcB-Algorithmus [8] ermittelt werden. Die Dauer (Anzahl der Abtastwerte) der für die Modellidentifikation genutzten Eingangssignale (Schallwandler- und Generatorsignal) ist dabei variabel, jedoch auf maximal 2500 Abtastwerte beschränkt. Dadurch ändert sich bei Vorhandensein eines Echos für jedes der erzeugten Modelle das zeitliche Verhältnis vom ungestörten Wandlersignal (Freifeldbedingung) zum Wandlersignal mit Echoeinfluss. Hierbei wird die Modellordnung des IIR-Filters möglichst gering gewählt (bei den vorliegenden Messungen: 16), um nur die Systemeigenschaften abzubilden und den Einfluss schwacher Empfangssignale auf das Modell zu minimieren. Zur Beurteilung der Modelle kann im Allgemeinen die Sendesignalunterdrückung herangezogen werden. Da bei einem Einsatz als Abstandssensor allerdings keine Freifeldmessungen vorliegen, kann dieses Kriterium nur für die Evaluation der Modelle unter Laborbedingungen offline genutzt werden. Für eine Online-Bewertung der Modelle ist ein anderes Kriterium notwendig, welches im folgenden Abschnitt Signalverarbeitung vorgestellt wird.

Für die weiteren Berechnungen erfolgt eine Bestimmung der Eigenschwingungen des modellierten Systems aus der berechneten Übertragungsfunktion $\mathrm{G}(\mathrm{z})$. Sie lassen sich aus deren Polstellen $P_{\mathrm{i}}$ und der bekannten Abtastzeit $t_{\mathrm{s}}$ mit (1) und (2) berechnen. Die beiden daraus gewonnenen Größen Kreisfrequenz $\omega_{i}$ und Dämpfung $d_{\mathrm{i}}$ der Eigenschwingungen werden im Folgenden als Eigenschwingungsparameter bezeichnet.

$$
\begin{gathered}
\omega_{i}=\frac{\arctan 2\left(\operatorname{imag}\left(P_{i}\right), \operatorname{real}\left(P_{i}\right)\right)}{t_{s}} \\
d_{i}=\frac{\log \left(\operatorname{abs}\left(P_{i}\right)\right)}{t_{s}}
\end{gathered}
$$

Nach der Zerlegung können die einzelnen Eigenschwingungen des Modells für die weitere Verarbeitung zu den einzelnen Eigenschwingungen des Schallwandlers zugeordnet werden. Dazu ist es allerdings notwendig, ungefähr die Wandlerresonanz- bzw. -antiresonanzfrequenz sowie die Dämpfung bei den Resonanzfrequenzen zu kennen (Startpunkte). Dann erfolgt die Zuordnung der Eigenschwingungen indem für alle möglichen Permutationen der Zuordnung der Gesamtabstand zu den Startpunkten minimiert wird. 


\section{Signalverarbeitung}

Für die Signalverarbeitung werden das digitalisierte Schallwandlersignal $u_{W}(k T)$ und das digitalisierte Generatorsignal $u_{\mathrm{G}}(k T)$ verwendet, wie in Abbildung 6 dargestellt. Um für die Schallausbreitung nicht relevante Resonanzfrequenzen des Schallwandlers zu unterdrücken, erfolgt zunächst eine Bandpassfilterung des Schallwandlersignals. Dadurch beeinflussen diese die folgende Systemidentifikation nicht, und es können kleinere Modellordnungen verwendet werden. Die Systemidentifikation wird hier für 21 verschieden lange Signalausschnitte durchgeführt. So werden 21 verschiedene Übertragungsfunktionen $\mathrm{G}_{n}(\mathrm{z})$ erzeugt. Anschließend erfolgt eine Bestimmung der Eigenschwingungsparameter (Dämpfung $d_{n i}$ und Kreisfrequenz $\omega_{n i}$ ) für jede Übertragungsfunktion. Die Übertragungsfunktionen sowie die Eigenschwingungsparameter werden dann an einen Auswahlalgorithmus übergeben, der daraus eine geeignete Übertragungsfunktion $G_{W}(z)$ für die Sendesignalkompensation auswählt, wie im nachfolgenden Abschnitt Auswertung genauer erläutert wird. Für die Sendesignalkompensation wird das geschätzte, gefilterte Schallwandlersignal $\tilde{W}_{\mathrm{WF}}(k T)$ mittels des Generatorsignals und des ausgewählten Modells berechnet. Dies wird von dem gemessenen und mittels Bandpass gefilterten Schallwandlersignal $u_{\mathrm{WF}}(k T)$ subtrahiert, um eine Schätzwert für das Empfangssignal $\tilde{u}_{E}(k T)$ zu erhalten.

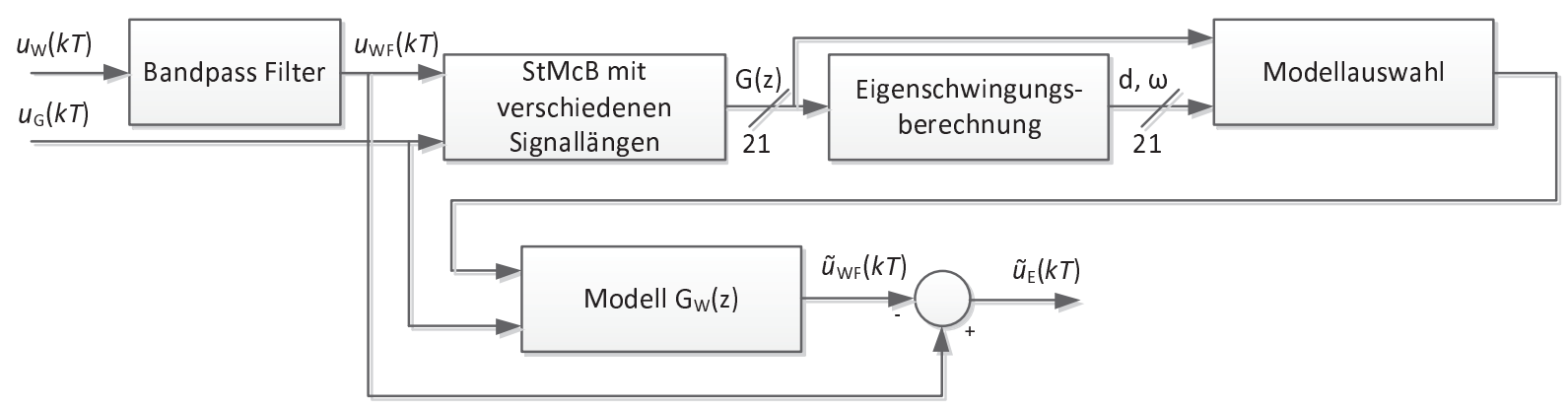

\section{Abbildung 6: Signalverarbeitungsschema für die Systemidentifikation, Schallwandler- und Empfangssignalberechnung}

\section{Auswertung}

Wenn bei der Modellbildung ein Echoanteil im Schallwandlersignal vorhanden ist, so wird dies im Modell mit abgebildet. Dadurch kommt es bei der Sendesignalkompensation zu einer Unterdrückung des Empfangssignals oder zu Phantomechos.

Um den Echoanteil im Schallwandlersignal abzuschätzen, werden für jede Messung Modelle aus unterschiedlich langen Abschnitten der gemessenen Schallwandler- und Generatorsignale bestimmt. Falls ein Echo im Signal vorhanden ist, variiert der Einfluss des Echos auf die Modelle entsprechend dem Verhältnis von (durch das Echo) gestörter zu ungestörter Signaldauer. Um diese auszuwerten, erfolgt eine Betrachtung der Frequenz und Dämpfung der Eigenschwingungen. Abbildung 7 zeigt die Eigenschwingungsparameter für zwei Messungen mit unterschiedlichen Reflektorabständen (anstelle der Kreisfrequenz $\omega$ ist hier die Frequenz $f$ dargestellt, um den Bezug zur Schallwandlerresonanz sichtbar zu machen). 

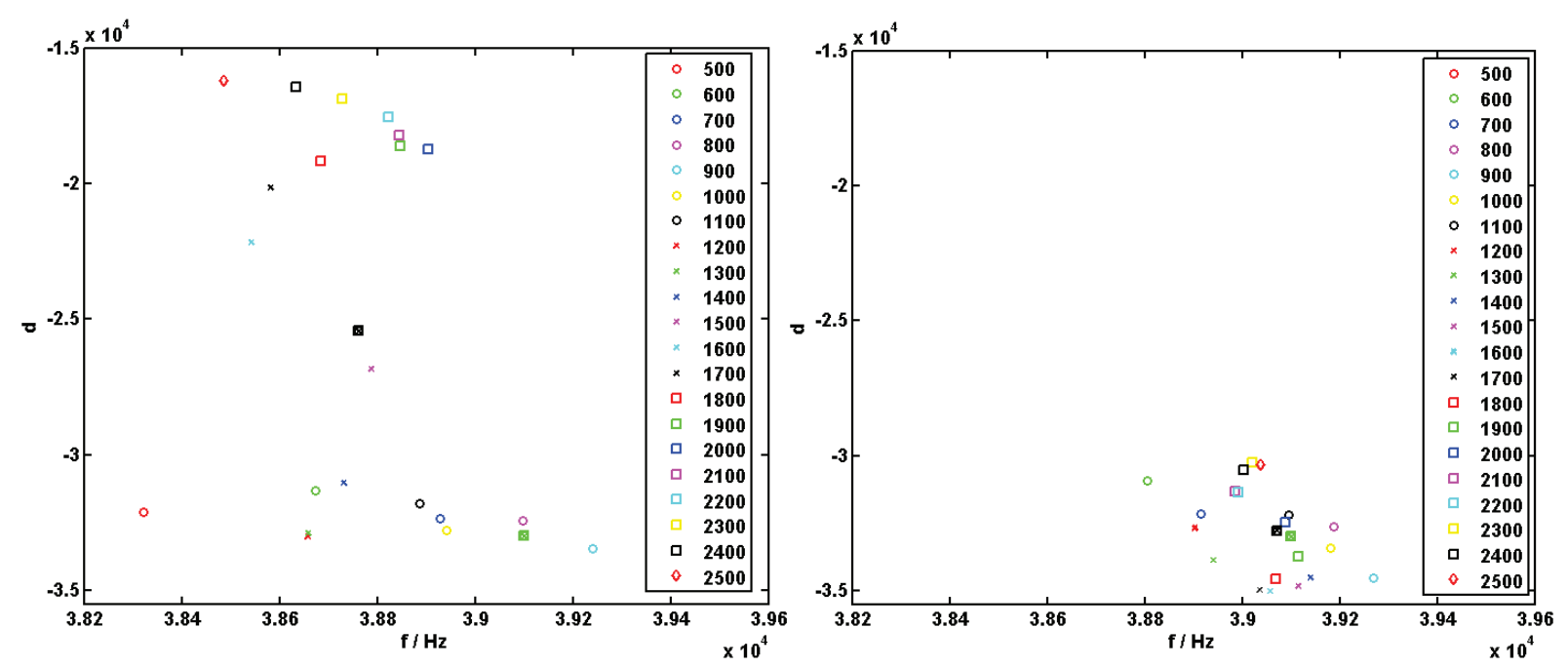

Abbildung 7: Eigenschwingungsparameter $\left(d_{n i}, \omega_{n i} b z w . d_{n i}, f_{n i}\right)$ für die Schallwandlerresonanz im Modell für verschiedene Signallängen (von 500 bis 2500 Abtastwerte) aus der

Systemidentifikation. Links bei einem Reflektorabstand von $50 \mathrm{~mm}$ und rechts bei $100 \mathrm{~mm}$

Zur Bewertung wird hier der geometrische Abstand der Eigenschwingungsparameter von dem Schwerpunkt aller Eigenschwingungsparameter genutzt. Hierbei ist gut zu erkennen, dass die Streuung der Eigenschwingungsparameter mit kleinerem Reflektorabstand (also größerem Echoanteil) zunimmt. Dieser Effekt kann genutzt werden, um die Güte des erzeugten Modells abzuschätzen. Der Verlauf der Streuung des Abstandes der Eigenschwingungsparameter von dem Schwerpunkt über den Reflektorabstand ist in Abbildung 8 dargestellt. Hier ist gut zu erkennen, dass der Echoeinfluss bei kleineren Abständen sehr stark ist und bei größeren Abständen nahezu konstant bleibt. Die Periodizität am Anfang entspricht der halben Wellenlänge des Sendesignals und scheint durch die unterschiedliche Phasenlage zwischen Sende- und Empfangssignal hervorgerufen zu werden.

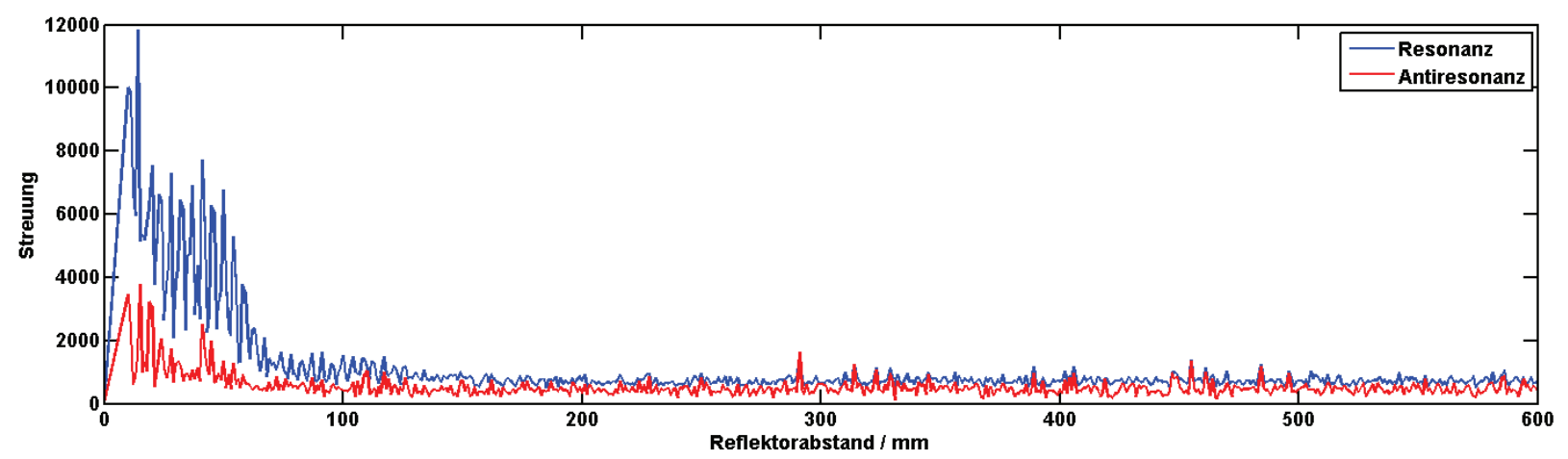

Abbildung 8: Verlauf der Streuung der Eigenschwingungsparameter für Resonanz und Antiresonanz des Wandlermodells in Abhängigkeit vom Reflektorabstand

Durch diese Bewertung der erzeugten Modelle ist es möglich, Modelle mit geringer

Sendesignalunterdrückung bei der weiteren Verarbeitung geringer zu gewichten oder ganz zu ignorieren. So liefert z.B. ein Modell, dass bei einem hohen Reflektorabstand erzeugt wurde, also einen geringen Echoanteil besitzt, für geringe Reflektorabstände eine höhere Sendesignalunterdrückung als ein Modell, welches bei einem geringeren Reflektorabstand berechnet wurde.

Um nach einer einzelnen Messung ein gültiges Modell zu erhalten, muss aus den 21 erzeugten Modellen, die bei unterschiedlichen Signallängen gewonnen worden sind und eine Modellgruppe bilden, ein Modell für die Sendesignalkompensation ausgewählt werden. Zur Bewertung dient hierbei der Abstand der Eigenschwingungsparameter von einem Referenz- oder Startpunkt. Dieser Referenzpunkt kann entweder fest definiert sein oder aus einer großen Anzahl von Messungen ermittelt werden. In Abbildung 9 sind die berechneten Schwerpunkte der ermittelten Modellgruppen für verschiedene Abstände aufgetragen. Hier zeigt sich deutlich eine Gruppierung der Eigenschwingungsparameter für Resonanz und Antiresonanz um einen Punkt. 


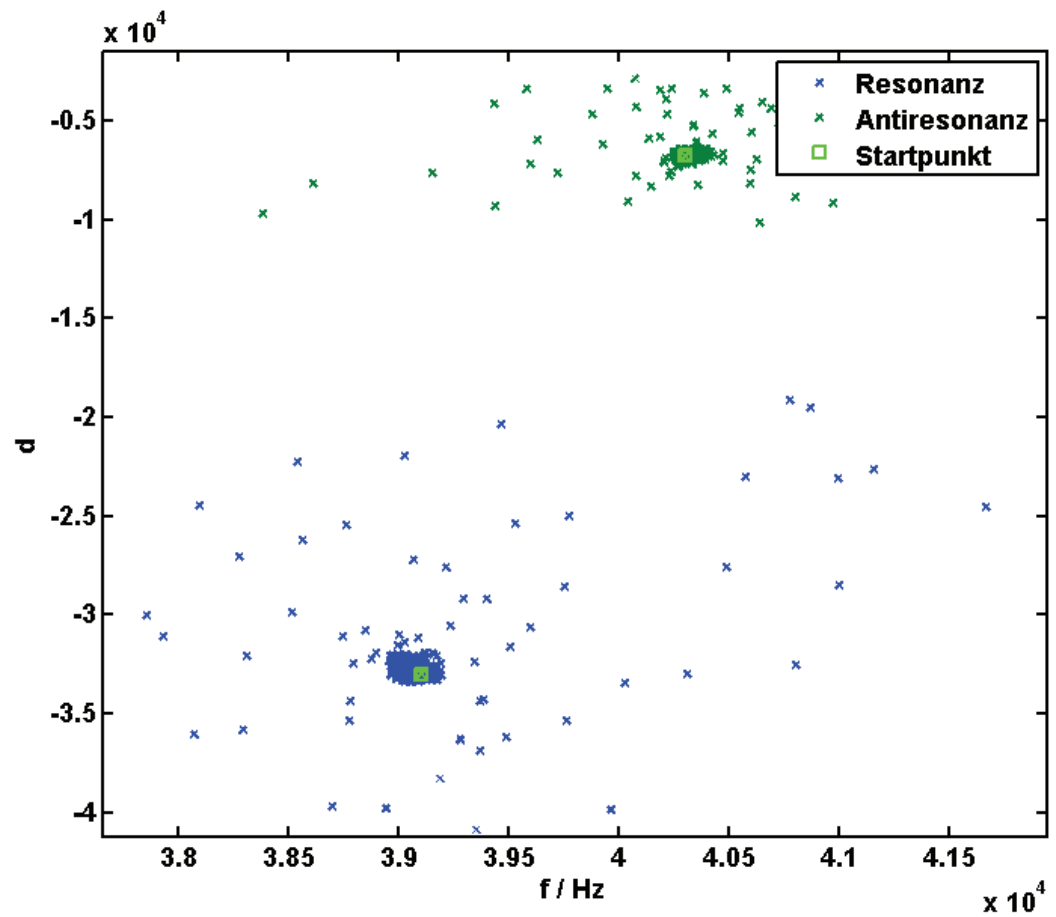

Abbildung 9: Schwerpunkte der Eigenschwingungsparameter für die Resonanz und Antiresonanz des Schallwandlermodells für 591 Messungen bei Reflektorabständen von $10 \mathrm{~mm}$ bis $600 \mathrm{~mm}$ (Schrittweite: $1 \mathrm{~mm}$ )

\section{Abstandsbestimmung}

Nach der Bestimmung des geschätzten Empfangssignals aus einer Messung kann der Abstand des Reflektors ermittelt werden. Dies erfolgt mittels einer Kreuzkorrelation zwischen dem berechneten Empfangssignal und dem Generatorsignal. Abbildung 10 zeigt die Einhüllenden der normierten Kreuzkorrelation für verschiedene Reflektorabstände. Hier ist der nahezu äquidistante Abstand der Maxima zu erkennen. Nur für sehr geringe Reflektorabstände (hier bei $10 \mathrm{~mm}$ (rot) zu erkennen) liefert eine einzelne Messung keine nutzbaren Ergebnisse.

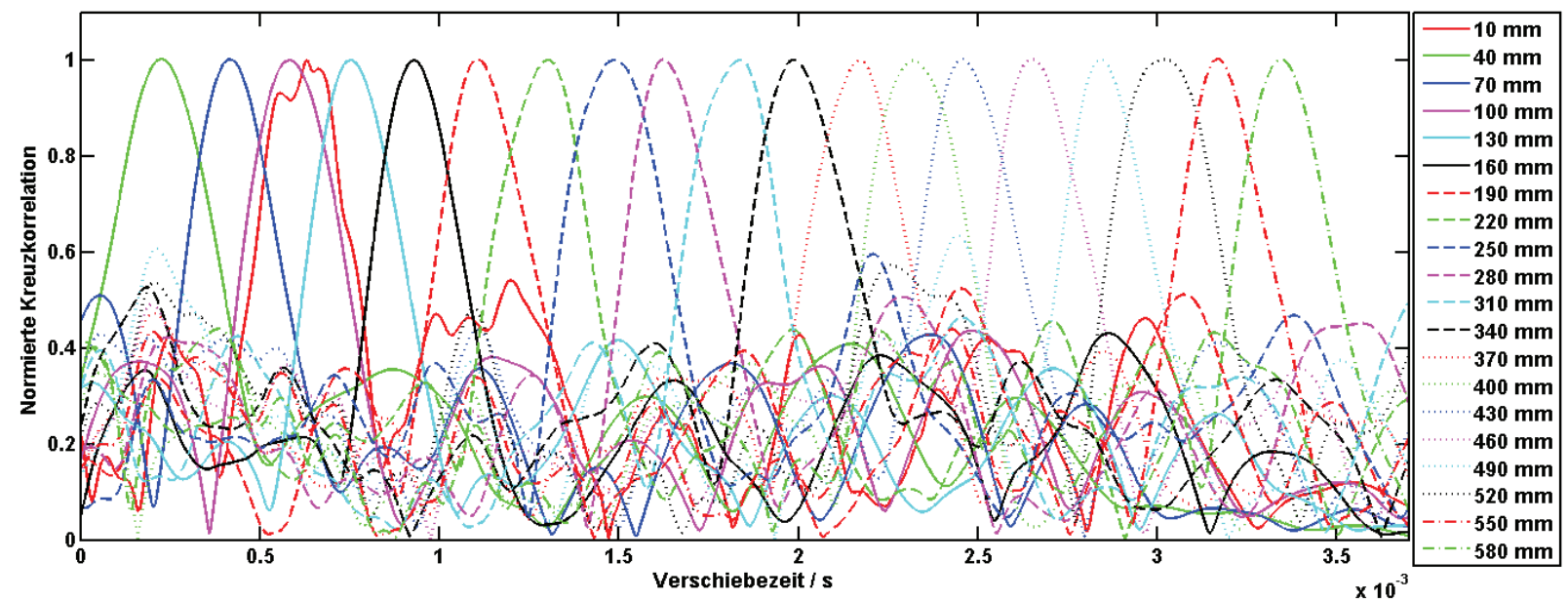

Abbildung 10: Einhüllende der normierten Kreuzkorrelation zwischen dem Generatorsignal und dem jeweils berechneten Empfangssignal für verschiedene Reflektorabstände

Die Abstandsmessung zeigt, wie in Abbildung 11 dargestellt, sehr große Abweichungen für Abstände unterhalb von $40 \mathrm{~mm}$. Hier ist der Echoanteil im Schallwandlersignal so groß, dass kein nutzbares Modell mehr erzeugt werden kann. Allerdings kann dieser Bereich vom System erkannt werden, denn hier ist die Streuung der Eigenschwingungsparameter, wie in Abbildung 8 dargestellt, sehr hoch. Hier ist es 
notwendig ein Modell, welches bei einer vorherigen Messung mit geringerer Streuung der Eigenschwingungsparameter ermittelt wurde, für die Kompensationssignal- bzw.

Empfangssignalberechnung zu verwenden.

Die in Abbildung 11 dargestellten absoluten Messabweichungen resultieren einerseits aus der Unvollkommenheit des Modells, weshalb keine vollständige Sendesignalunterdrückung gelingt. Eine hierdurch verursachte Phasenverschiebung des berechneten Empfangssignals, verschiebt somit auch das Maximum der Kreuzkorrelation. Zu erkennen ist dies an der Periodizität der Messabweichungen, die gerade einer halben Wellenlänge des Sendesignals entspricht. Andererseits zeigt der Verlauf der Messabweichungen einen geringen Offsetversatz, der auf eine bisher nicht exakt korrigierte Phasenverschiebung des Schallwandlersignals durch den Bandpass-Filter zurückzuführen ist. Bei einem Abstand zwischen $400 \mathrm{~mm}$ und $500 \mathrm{~mm}$ zeigt sich ebenfalls eine größere Messabweichung. Hier liegt die Ursache wiederum in der begrenzten Sendesignalunterdrückung. Oberhalb eines Abstandes von $500 \mathrm{~mm}$ ist dieser Einfluss deutlich geringer, da hier ein Großteil des Empfangssignals nach dem Ausschwingen des Schallwandlers eintrifft und somit ohne Kompensation fehlerfrei erfasst werden kann. Des weiteren ist die Auflösung bei der Abstandsmessung durch die geringe Bandbreite und die daraus resultierenden breiten Maxima der Kreuzkorrelation begrenzt.

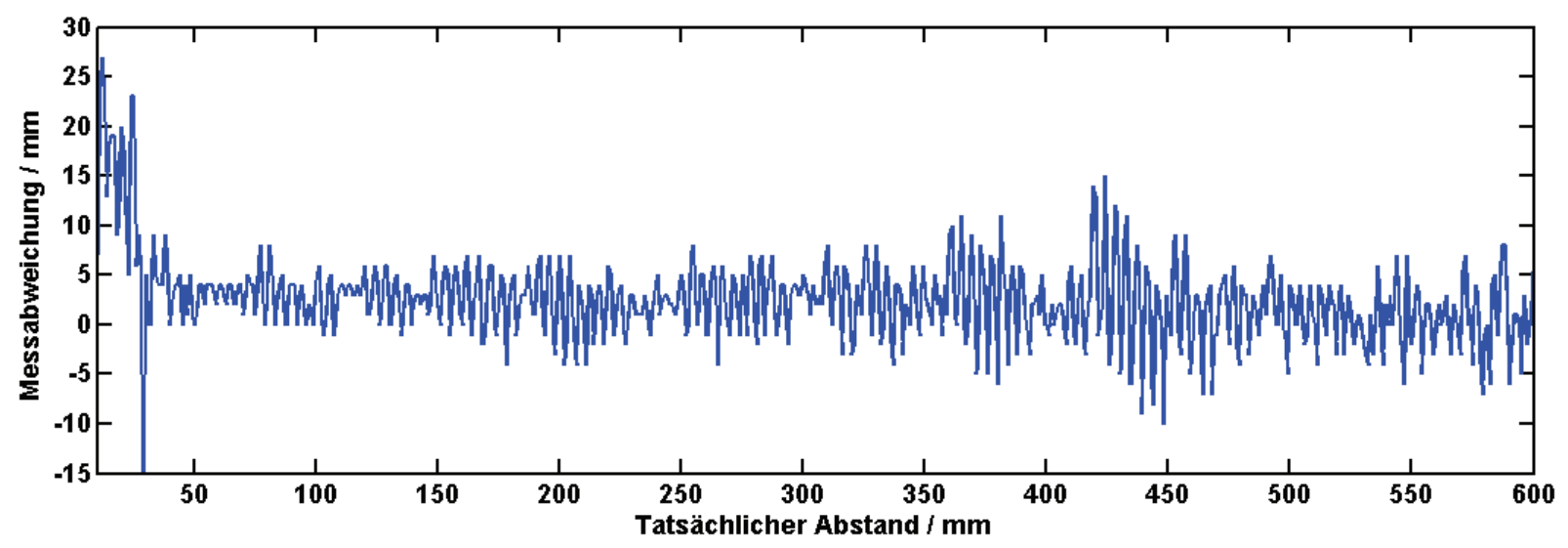

Abbildung 11: Absolute Messabweichung des berechneten Reflektorabstands

\section{Zusammenfassung und Ausblick}

Mit dem vorgestellten Verfahren ist es möglich, die Stabilität eines modellbasierten gleichzeitigen Sendeund Empfangsbetriebes durch die Bewertung der aus Einzelmessungen erzeugten Modelle zu erhöhen. Dies kann unter anderem dazu genutzt werden, eine blindzonenfreie Abstandsmessung zu realisieren. Da die hier genutzten Modelle aus Einzelmessungen erzeugt werden, ist es möglich, Messungen direkt nach dem Einschalten des Systems und der Durchführung einer Auto-Parametrierung durchzuführen. Nur bei sehr geringen Abständen unterhalb von 40 mm (etwa 4 Wellenlängen) kommt es zu größeren Messabweichungen, die allerdings durch die Bewertung der Modelle über die Streuung der Eigenschwingungsparameter erkannt werden können. Sehr geringe Abstände können daher erst nach mehreren Messungen erfasst werden, nachdem ein gültiges Modell ermittelt wurde.

Für die Implementierung des Verfahrens in einem Mikrocontroller- oder DSP-basierten Sensor ist eine Optimierung der verwendeten Algorithmen sinnvoll, da die relativ hohe Anzahl von Abtastwerten, die für die Systemidentifikation genutzt werden, zu großen Matrizen führt, die eine große Anzahl von Rechenoperationen und viel Speicherplatz benötigen. Eine Möglichkeit diesen Rechenaufwand zu reduzieren, ist die geringe Bandbreite des Schallwandlers auszunutzen und eine Bandpassunterabtastung zu verwenden.

Eine Verringerung der Messabweichungen kann zum einen durch eine Verbesserung der Sendesignalunterdrückung mittels eines iterativen Modellierungsverfahrens oder zum anderen durch eine Verbesserung der Empfangssignalauswertung erzielt werden. Hierbei bietet sich z.B. eine künstliche Erhöhung der Bandbreite des Schallwandlers durch einen Gyrator an, um die Korrelationseigenschaften des Empfangssignals zu verbessern.

\section{Literatur}

[1] R. Lerch, G. Sessler, D. Wolf, "Technische Akustik”, Springer Berlin Heidelberg, 2009, P. 35 - 53, ISBN: 978-3-540-49833-9 
[2] G. Bradfield, "Improvements in Ultrasonic Flaw Detection“, Journal of the British Institute of Radio Engineers 14 (1954) P. $303-308$

[3] T. Vössing, J. Rautenberg, R. Kehl, B. Henning, "Simultaneous Transmitting and Receiving with Ultrasonic Sensors", $13^{\text {th }}$ International Conference Sensor + Test 2007, Nuremberg, 22.05. 24.05.2007, Proceedings Volume II A8.1, P. 69 - 74 (ISBN: 978-3-9810993-2-4)

[4] A. Schröder, C. Hoof, B. Henning, „Ultrasonic Transducer Interfacecircuit for Simultaneous Transmitting and Receiving", ICEMI '2009, Beijing, China, Proceedings Vol. 4 (ISBN: 978-1-42443862-4)

[5] A. Schröder, B. Henning, Interfaceschaltung für einen simultanen Sende- und Empfangsbetrieb mit einem einzelnen Ultraschallwandler. In 36. Jahrestagung für Akustik (DAGA 2010), Berlin, 15.-18.03.2010 , pp. 1025-1026 (2010)

[6] A. Schröder, B. Henning, "Improved system identification for simultaneous transmitting and receiving in single transducer applications". In IEEE IUS 2010, San Diego, California, USA, 11.14.10.2010

[7] F. S. Granja, A. R. Jim'enez, "Ultrasound modulation and codification for localization systems", In New Acoustics: selected topics II, 2006, P. 167-186

[8] K. Steiglitz, L. E. McBride, "A Technique for the Identification of Linear Systems," IEEE Transactions on Automatic Control, 4 (October 1965), P. $461-465$ 